\title{
A Descriptive Analysis of the Hip and Knee Joint Replacement Procedures of the University of the Philippines - Philippine General Hospital (UP-PGH) from 2012 to 2018
}

\author{
Jose Carlos. F. Dumlao, MD, Giorgio D. Delgado, MD and Gregorio Marcelo S. Azores, MD \\ Arthroplasty Service, Department of Orthopedics, Philippine General Hospital, University of the Philippines Manila
}

\begin{abstract}
Objective. Hip and knee joint replacement procedures are an effective therapeutic intervention in treating severe joint disorders. Its use has been increasingly performed worldwide, including the Philippines, with its techniques constantly evolving and the science behind it improving. This study aims to describe the demographics, clinical profiles, and outcomes of arthroplasty patients by the Arthroplasty Service, Department of Orthopedics, University of the Philippines - Philippine General Hospital (UP-PGH).
\end{abstract}

Methods. The study is a descriptive and retrospective review of patients who underwent joint replacement procedures, both primary and revision arthroplasty, from January 2012 to December 2018. Patient demographics and clinical data of patients who underwent total joint arthroplasty at the UP-PGH were collected and evaluated.

Result. Data from 279 patients with 306 primary joint replacement procedures were analyzed. There were 195 total hip arthroplasty procedures (THAs) and 111 total knee arthroplasty procedures (TKAs) done. The mean age for THA patients was 55.6 years old, with more females (68.2\%) with the left hip being more commonly affected (54.9\%). The most common indication for THA was an untreated femoral neck fracture (23.1\%) followed by avascular necrosis (20.5\%). Cementless fixation was the most commonly used technique (61.5\%). Meanwhile, the mean age for TKA was 64.5 years old, with the majority having degenerative osteoarthritis, and using cemented TKA fixation for all knees. A total of 37 revision arthroplasty cases were performed, with 34 in the hip and three in the knee, with infection being the most common overall indication (53\%).

Conclusion. The demographics, clinical profiles, and outcomes of the UP-PGH Arthroplasty Service are comparable to other centers internationally, and further emphasizes the satisfactory outcomes of these procedures. Meanwhile, suggested explanations for the subtle differences are discussed in this study.

Key Words: descriptive analysis, clinical profile, outcomes, total hip arthroplasty, total knee arthroplasty, revision arthroplasty

\section{INTRODUCTION}

Corresponding author: Gregorio Marcelo S. Azores, MD Chief, Arthroplasty Service

Department of Orthopedics

Philippine General Hospital

University of the Philippines Manila

Taft Avenue, Ermita, Manila 1000, Philippines

Email: gsazores@up.edu.ph
Total joint replacement or arthroplasty of the hips and knees is a well-established orthopedic surgical procedure that has been among the most successful forms of surgeries especially within the past five decades., ${ }^{1,2}$ Due to the improvements in surgical technique and implant design and longevity, arthroplasty has increasingly been performed. ${ }^{3}$

Osteoarthritis (OA) is the leading cause of pain and disability worldwide. ${ }^{4}$ It is estimated that osteoarthritis is the fourth leading cause of disability. ${ }^{5} \mathrm{OA}$ is the indication for more than $90 \%$ of the increasing number of total hip or knee joint replacement operations being undertaken around the globe. ${ }^{6}$ Its success in the treatment of OA has 
led to the increasing demand for total hip arthroplasty and total knee arthroplasty. ${ }^{7}$ In the United States, it is estimated that there were 168,000 discharges among individuals aged above 65 years for total hip arthroplasty in $2010^{8}$ and around 500,000 total knee arthroplasty procedures for 2009. ${ }^{9}$ Other indications for joint replacement procedures include avascular necrosis, inflammatory arthritis, complicated fractures, and a multitude of other joint disorders.

Hip and knee arthroplasty procedures have entered mainstream clinical practice in the Philippines for the given indications. The Department of Orthopedics, UP-PGH has been performing and is continuously refining its techniques for the past decades. To analyze its data and monitor its outcomes, and to serve as baseline data for future researches, an analysis of its census was conceived. This study aims to describe the patient demographics, clinical profiles, and outcomes of patients who underwent primary or revision arthroplasty procedures of the hip and knee joints in the UP-Philippine General Hospital from 2012 to 2018.

\section{MATERIALS AND METHODS}

Patients who underwent primary and revision total joint arthroplasty of the hip and knee from January 2012 to December 2018 at the University of the Philippines Philippine General Hospital (UP-PGH) were included in this study. These include cases ranging from femoral neck fractures, post-traumatic arthritis, degenerative and inflammatory joint conditions. Patients were either handled or supervised by consultants of the Arthroplasty Service and Trauma Service of the Department of Orthopedics. Patients who underwent partial hip arthroplasty or hemiarthroplasty were not included as well as musculoskeletal oncology patients who had an arthroplasty for their operation.

This study obtained its Ethics Review Board approval from the hospital. A chart review method was performed for all patients, taking note of details such as age, sex, diagnosis, laterality, the procedure performed, kind of fixation, and complications. The study used frequency and percentage analysis to know the number of the different variables. Descriptive analysis was used for the characteristics of the population of the study.

\section{RESULTS}

The study population consisted of 306 primary total joint arthroplasties from 279 patients; 195 for total hip arthroplasties (THAs) and 111 for total knee arthroplasty operations (TKAs). A total of 37 revision arthroplasty surgeries were done; 34 for hip revision surgeries and 3 for knee revision surgeries.

The mean age of patients undergoing total hip arthroplasty was 55.6 years old (range $19-88$ years) and 64.5 years old for patients undergoing total knee arthroplasty (range $32-85$ years). Table 1 shows the demographic data of
Table 1. Demographic data of primary total hip arthroplasty patients

\begin{tabular}{lc}
\multicolumn{1}{c}{ Demographic } \\
Sex \\
Male n (\%) \\
Female $\mathrm{n}(\%)$ & $62(31.8 \%)$ \\
\hline Age & $133(68.2 \%)$ \\
Mean & 55.6 \\
Mode & 57 \\
Median & 57 \\
Range & $19-88$ \\
\hline Laterality & \\
Right & $88(45.1 \%)$ \\
Left & $107(54.9 \%)$ \\
\hline Fixation & \\
Cementless & $120(61.5 \%)$ \\
Cemented & $70(35.9 \%)$ \\
Hybrid & $5(2.6 \%)$ \\
Reverse Hybrid & $0(0 \%)$ \\
\hline Primary Diagnosis n (\%) & \\
Untreated Femoral Neck & $45(23.1 \%)$ \\
Avascular Necrosis & $40(20.5 \%)$ \\
Femoral Neck Fracture & $29(14.9 \%)$ \\
Dysplastic Hip & $26(13.3 \%)$ \\
Degenerative Osteoarthritis & $19(9.7 \%)$ \\
Pathologic Fracture & $8(4.1 \%)$ \\
TB arthritis & $6(3.1 \%)$ \\
Juvenile Arthritis & $4(2.1 \%)$ \\
Neglected SCFE & $4(2.1 \%)$ \\
Rheumatoid Arthritis & $4(2.1 \%)$ \\
Ankylosing spondylitis & $3(1.5 \%)$ \\
Posttraumatic Arthritis & $2(1.0 \%)$ \\
Untreated dislocation & $2(1.0 \%)$ \\
Nmplant failure & $1(0.5 \%)$ \\
Untreated intertrochanteric fracture & $1(0.5 \%)$ \\
\hline & $1(0.5 \%)$ \\
\hline
\end{tabular}

total hip arthroplasty patients. Most patients are of the female sex (68.2\%) and the left hip is commonly affected (54.9\%). The majority of fixation for hip arthroplasty surgeries is the cementless fixation (61.5\%) followed by cemented fixation (35.9\%) and hybrid fixation (2.6\%). The most common primary diagnosis indicated for total hip arthroplasty would be an untreated femoral neck fracture (23.1\%) followed by avascular necrosis (20.5\%), acute femoral neck fractures (14.9\%), and dysplasia of the hips (13.3\%).

Table 2 shows the demographic profile of total knee arthroplasty patients. A total of 111 total knee arthroplasty operations were done on 101 patients, most of whom were female (83.8\%). All of the TKA surgeries were done using cemented fixation. The most common indication for TKA is degenerative osteoarthritis $(86.5 \%, \mathrm{n}=96)$ followed by rheumatoid arthritis $(11.7 \%, \mathrm{n}=13)$.

The $<55$ age group has the most number of procedures for total hip arthroplasty and is followed by the 56-65 and 
Table 2. Demographic data of primary total knee arthroplasty patients

\section{Demographic}

\begin{tabular}{lc} 
Sex & \\
Male n (\%) & $18(16.2 \%)$ \\
Female n (\%) & $93(83.9 \%)$ \\
\hline Age & \\
Mean & 64.5 \\
Mode & 63 \\
Median & 66 \\
Range & $32-85$ \\
\hline Laterality & \\
Right & $59(53.2 \%)$ \\
Left & $52(46.8 \%)$ \\
\hline Fixation & \\
Cementless & $0(0 \%)$ \\
Cemented & $111(100.0 \%)$ \\
\hline Primary Diagnosis $n$ (\%) & \\
Degenerative Osteoarthritis & $96(86.5 \%)$ \\
Rheumatoid Arthritis & $13(11.7 \%)$ \\
Psoriatic Arthritis & $1(0.9 \%)$ \\
TB Arthritis & $1(0.9 \%)$ \\
\hline
\end{tabular}

Table 3. Kind of fixation according to age group

\begin{tabular}{lcccrr}
\multicolumn{1}{c}{ Fixation } & Cementless & Cemented & Hybrid & Total & (\%) \\
Age Group & & & & & \\
$\quad<55$ & 75 & 10 & 3 & 88 & 45 \\
$56-65$ & 33 & 10 & 2 & 45 & 23 \\
$66-75$ & 12 & 36 & & 48 & 25 \\
$>75$ & & 14 & & 14 & 7 \\
Total & 120 & 70 & 5 & 195 & \\
Percentage (\%) & 62 & 36 & 2 & & \\
\hline
\end{tabular}

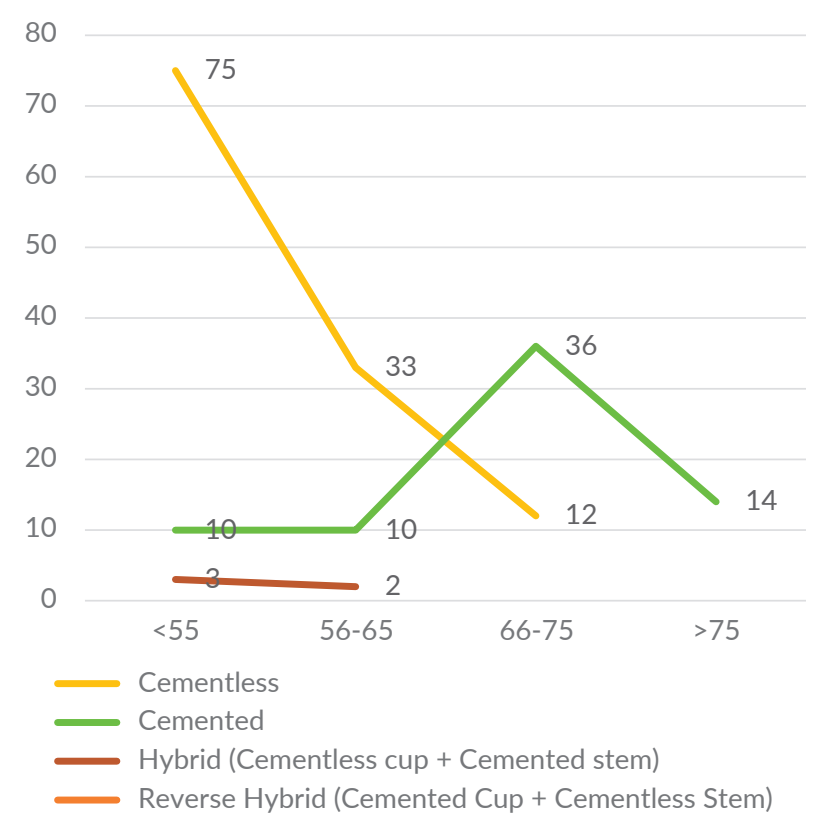

Figure 1. Kind of fixation by age group.
Table 4. Percentage of diagnosis by age group

\begin{tabular}{|c|c|c|}
\hline Diagnosis & $\mathbf{n}$ & (\%) \\
\hline \multicolumn{3}{|l|}{$<55$} \\
\hline AVN & 34 & 39 \\
\hline Dysplastic Hip & 15 & 17 \\
\hline Untreated femoral neck fracture & 8 & 10 \\
\hline Juvenile Arthritis & 4 & 5 \\
\hline Rheumatoid Arthritis & 4 & 5 \\
\hline TB Arthritis & 4 & 5 \\
\hline Ankylosing Spondylitis & 3 & 3 \\
\hline Degenerative OA & 3 & 3 \\
\hline Fracture femoral neck & 3 & 3 \\
\hline Pathologic fracture & 3 & 3 \\
\hline Degenerative OA (LCPD) & 2 & 2 \\
\hline Untreated dislocation & 2 & 2 \\
\hline Neglected SCFE & 1 & 1 \\
\hline Nonunion & 1 & 1 \\
\hline Posttraumatic Arthritis & 1 & 1 \\
\hline \multicolumn{3}{|l|}{$56-65$} \\
\hline Dysplastic Hip & 10 & 22 \\
\hline Fracture femoral neck & 10 & 22 \\
\hline Degenerative OA & 8 & 18 \\
\hline Untreated femoral neck fracture & 8 & 18 \\
\hline Neglected SCFE & 3 & 8 \\
\hline AVN & 2 & 4 \\
\hline Pathologic fracture & 2 & 4 \\
\hline TB Arthritis & 2 & 4 \\
\hline \multicolumn{3}{|l|}{$66-75$} \\
\hline Untreated femoral neck fracture & 21 & 44 \\
\hline Fracture femoral neck & 13 & 28 \\
\hline Degenerative OA & 5 & 10 \\
\hline AVN & 4 & 8 \\
\hline Pathologic fracture & 2 & 4 \\
\hline Dysplastic Hip & 1 & 2 \\
\hline Posttraumatic Arthritis & 1 & 2 \\
\hline Untreated intertrochanteric femur & 1 & 2 \\
\hline \multicolumn{3}{|l|}{$>75$} \\
\hline Untreated femoral neck fracture & 8 & 57 \\
\hline Fracture femoral neck & 3 & 22 \\
\hline Degenerative OA & 1 & 7 \\
\hline Implant Failure & 1 & 7 \\
\hline Pathologic fracture & 1 & 7 \\
\hline
\end{tabular}

66-75 age groups with a similar percentage $(23 \%, 25 \%)$. Sixty-two percent (62\%) of the THA patients have a cementless fixation, most of which are from the $<55$ age group. Cemented fixation is mostly from the 66-75 and $>75$ age group comprising almost 70 percent of all cemented total hip arthroplasty. Only 2 percent of all total hip arthroplasty operations used hybrid fixation. Table 3 shows a kind of fixation by age group and Figure 1 shows the graph of fixation by age group.

Table 4 shows the distribution of diagnosis by age group. On the $<55$ age group, the top 3 diagnoses were avascular necrosis (39\%), dysplastic hips (17\%), and untreated femoral 
neck fractures (10\%). Following the 56-65, and 65-74 age group, the top diagnosis was hip dysplasia, acute and untreated femoral neck fracture, and degenerative osteoarthritis. Lastly, for the $>75$ age group, more than $50 \%$ are from the untreated femoral neck fracture.

\section{Revision cases}

A total of 37 cases of total joint revision surgeries were recorded (Table 5). For the revision surgeries, 34 cases were hip (91\%) and 3 cases (9\%) are of knee revision surgeries.

The most common indication for revision hip surgeries was infection (53\%) followed by dislocation (20\%) then due to aseptic loosening/osteolysis (18\%). Of the 18 infections, 4 patients proceeded with two-stage revision surgery. There is one mortality due to pneumonia, 3 underwent hanging hip procedures and six underwent 1 -stage revision then were lost to follow up. Two out of the seven dislocations needed open reduction with revision of implants, of which one case needed acetabular component revision and the other needed a femoral stem revision.

All knee revision surgeries were due to infection in which one underwent 2-stage revision and the other two underwent the 1-stage procedure.

Table 6 shows the complication rate of total hip arthroplasty patients at the UP-PGH 2012 - 2018. Out of all the 18 infections in 14 patients, the index procedure of

Table 5. Indication of revision hip and knee surgeries

\begin{tabular}{lrc}
\hline Cases of Revision Surgeries (Hip and Knee) & 37 & \\
Hip revision surgeries & 34 & $92 \%$ \\
Knee revision surgeries & 3 & $8 \%$ \\
\hline \multicolumn{1}{c}{ Breakdown } & $\mathbf{n}$ & $\mathbf{( \% )}$ \\
\hline Hip revision & & \\
$\quad$ Infections & 18 & $53 \%$ \\
Aseptic Loosening & 6 & $18 \%$ \\
Trauma/Prosthetic Fracture & 2 & $6 \%$ \\
Foreign Body & 1 & $3 \%$ \\
Dislocation & 7 & $20 \%$ \\
$\quad$ Closed reduction & 5 & \\
$\quad$ Open reduction & 2 & \\
Infections & & \\
1st stage & 14 & \\
2nd stage & 4 & \\
$\quad$ Hanging hip procedure & 3 & \\
\hline Knee revision & & \\
Infection & 3 & $100 \%$ \\
\hline
\end{tabular}

Table 6. The complication rate of In-House total hip arthroplasty patients in UP-PGH 2012-2018

\begin{tabular}{lcc} 
Primary and revision total hip arthroplasty (227) & (n) & Rate \\
Dislocation & 5 & $2.2 \%$ \\
Infection & 3 & $1.3 \%$ \\
Trauma/Prosthetic Fracture & 1 & $0.4 \%$ \\
Foreign Body & 1 & $0.4 \%$ \\
\hline
\end{tabular}

the 3 patients was done at our institution which happened within the study date which yielded a $1.3 \%$ infection rate. All of the other infections were done outside our institution or were done not within the study date. Of the 7 dislocations, 5 cases were done at UP-PGH and the computed dislocation rate of the institution is at $2.2 \%$.

\section{DISCUSSION}

The mean age of patients undergoing total hip arthroplasty by other researchers varied between 60.5 and 70 years old, ${ }^{1,2}$ this is above the mean age of this study of 55.6 years old. In total knee arthroplasty, our study revealed a mean of 64.5 years old of patients which is similar to the literature..$^{3-5}$ Osteoarthritis is still the leading diagnosis and indication for total hip arthroplasty or hip replacement., ${ }^{6,7}$ In the 2019 Annual Report of AOAJNRR, ${ }^{6}$ osteoarthritis is the most common diagnosis for hip replacement, followed by femoral neck fracture then osteonecrosis. In contrast, our study showed untreated femoral neck fracture (fractures $>28$ days old) as the most common diagnosis for total hip replacement. This is followed by avascular necrosis then acute femoral neck fractures. Untreated femoral neck fractures are common in developing countries because most patients do not seek treatment because of the non-availability of the hospital near their location. Most of the patients would also go to bonesetters or osteopaths for medical care. Also, with the financial burden of developing countries and the high cost of implants, most patients cannot afford the procedure. ${ }^{8}$ The UP-PGH is a tertiary hospital and a referral center for these kinds of patients. This may be the reason for the increase of untreated femoral neck fractures treated in our institution. Also, in terms of priority on the list of operations, these untreated femoral neck fractures are given more priority compared to the arthritic patients as they are more impaired in function. Osteoarthritis is the $5^{\text {th }}$ diagnosis for total hip replacement in this study. Degenerative osteoarthritis of the knees is the main indication for total knee arthroplasty and this is consistent with the literature. ${ }^{6,7}$

Female gender is still the most common in total hip arthroplasty and total knee arthroplasty according to previous studies and our study is consistent with these findings. ${ }^{8-11}$ In terms of the kind of fixation for THA, younger patients were likely to receive cementless kind of fixation. This is most likely attributed to the age and bone stock of younger patients. The use of hybrid fixation was not as popular an option for the patients who underwent THA.

As for those who underwent total hip arthroplasty, infection was the most common cause for revision. This is the same issue noted in other studies ${ }^{12-14}$ as well, but based on the Australian Registry ${ }^{6}$, other causes included loosening, prosthesis dislocation, with infection being the last. In New Zealand, they also note that the common cause for revision arthroplasty is dislocation. In their knee arthroplasties, their most common cause of revision is pain, followed by 
infection. ${ }^{15}$ In Canada, same with the Philippines, infection is the primary cause for revision as well, especially in cases of knee arthroplasty. For their total hip arthroplasty, however, aseptic loosening is the primary cause for revision, followed by instability and lastly by infection. ${ }^{16}$

These results imply the need to improve infection control measures in hospitals, especially in the Philippines where the most common cause for revision is infection. The infection may however be attributed to post-surgical, postdischarge infection, which also implies the need to educate the patient about infection control measures he/she needs to take upon discharge. In other countries, especially more advanced western countries, infection is less likely the cause for revision. This may be attributed to comparatively better infection control measures in their hospitals as compared to Philippine hospitals. Likewise, there may be inadequate follow-up from patients for other types of sequelae or complications, which may be giving infection an artificially high incidence. Thereby, postoperative surveillance for the other types of complications might need improvement in the Philippine healthcare setting.

Hip infection burden is defined as the ratio of hip arthroplasty implants revised for infection to the total number of arthroplasties in a specific period. ${ }^{17}$ Our study surveyed our hospitals' arthroplasty census to determine the burden of revision and complication rate. Our hip infection burden is computed at $1.7 \%$. For the knee infection burden, no infection was recorded in our institution from 2012 2018 for total knee replacement. Although data showed that 3 surgeries were done due to infection, the index cases were done outside the institution or not within the time frame of this study, thus, not included in the computation for infection burden. The computed hip burden infection is comparable to the reported $0.3 \%-1.7 \%$ rate in centers worldwide. ${ }^{13,14}$

The hip dislocation rate computed in this study is $2.2 \%$ which is high compared to international rates ranging from $0.5 \%$ to $1.5 \% .^{18}$ Reasons for dislocation would be poor soft tissue tension, component malposition which led to two open reductions with component replacement, polyethylene wear, and poor hip dislocation precautions.

This study is without its limitations. The cross-sectional design of the study cannot generate a temporal relationship between exposure and outcome. Characteristics or factors of the revision population may not provide precise data and conclusions due to the study design. The population of the study covers only one hospital but is nevertheless a tertiary hospital that uniquely caters to a population with a predominant lower socioeconomic status. As such, the results from this study cannot be generalized to the Metro Manila area. More factors in the demographic profile and surgical profile of these patients should also be considered such as comorbidities, functional outcomes, length of hospital stay, type of anesthesia, ASA classification, and BMI. Although a chart review was done for patients, not all patients had the complete information needed to make the analysis comprehensive. For example, the bearing surfaces used for the total hip arthroplasty should have also been included. A more comprehensive system to document all the parameters should be implemented to have a broader profile of arthroplasty patients. Lastly, given the limited data retrieved from the existing records, there are incomplete details especially those beyond the study period which likewise give us a limited analysis.

Due to incomplete follow-up data of all implanted patients during this period, the authors were unable to compute for the implant survivorship in our center for this cohort. This study recommends a more robust database with a more intensive follow-up for future studies, that may include a survivorship analysis.

\section{CONCLUSION}

This current database shows us that some parameters of the patient demographics, clinical profiles, and outcomes of the UP-PGH Arthroplasty Service were comparable to other centers internationally. With a deeper understanding of the possible reasons for the points of differentiation, as well as taking note of the areas of improvement of our census, a better analysis of the data and better management may be provided in the future.

\section{Statement of Authorship}

All authors participated in the data collection and analysis and approved the final version submitted.

\section{Author Disclosure}

All authors declared no conflicts of interest.

\section{Funding Source}

No funding support.

\section{REFERENCES}

1. Pachore JA, Vaidya SV, Thakkar CJ, Bhalodia HP, Wakankar HM. ISHKS joint registry: A preliminary report. Indian J Orthop 2013;47:505-9.

2. Total hip arthroplasty: Techniques and results. BCMJ, Vol. 52, No. 9, November, 2010, Page(s) 455-64 - Clinical Articles.

3. Daniel H. Williams, MSc, FRCS (Tr \& Orth),, Donald S. Garbuz, MD, MPH, FRCSC, Bassam A. Masri, MD, FRCSC. Total knee arthroplasty: Techniques and results. BCMJ, Vol. 52, No. 9, November, 2010, Page(s) 447-54 - Clinical Articles.

4. World Health Organization (2002) World Health Report 2002. Reducing Risks, Promoting Healthy Life. Geneva, WHO

5. Fransen M, Bridgett L, March L, Hoy D, Penserga E, Brooks P. (2011) The epidemiology of osteoarthritis in Asia. Int J Rheum Dis $14,113-21$.

6. Australian Orthopaedic Association (2018) Hip and Knee Arthoplasty. National Joint Replacement Registry Annual Report 2018

7. Franklin, P.D., Miozzari, H., Christofilopoulos, P, Hoffmeyer, P, Ayers, D, Lübbeke, A. Important patient characteristics differ prior to total knee arthroplasty and total hip arthroplasty between Switzerland and the United States. BMC Musculoskelet Disord 18, 14 (2017). https://doi.org/10.1186/s12891-016-1372-5 
8. Wu N, Chen SY, Lee YC, Zhao Y. Demographics and clinical and economic characteristics of patients receiving total hip arthroplasty with and without muscle atrophy/weakness. Clinicoecon Outcomes Res. 2013;5:271-280. Published 2013 Jun 25. doi:10.2147/CEOR. S46332

9. Cram P, Lu X, Kates SL, Singh JA, Li Y, Wolf BR. Total knee arthroplasty volume, utilization, and outcomes among Medicare beneficiaries, 1991-2010. JAMA. 2012;308(12):1227-36.

10. Goveia, Vania Regina, Mendoza, Isabel Yovana Quispe, Couto, Bráulio Roberto Gonçalves Marinho, Ferreira, Jose Antonio Guimarães, Paiva, Edson Barreto et al. Profile of hip arthroplasty patients in a teaching hospital. Rev. Col. Bras. Cir. [online]. 2015, vol.42, n.2 [cited 2019-11-27], pp.106-110. Available from: $<$ http://www.scielo.br/scielo.php?script=sci_arttext\&pid=S010069912015000300106\&lng=en\&nrm=iso >. ISSN 0100-6991. http:// dx.doi.org/10.1590/0100-69912015002007.

11. Wolfovitch LA (2017) Clinical and epidemiological profile of patients undergoing total hip arthroplasty. Rheumatol Orthop Med 2: DOI: 10.15761/ROM.1000120

12. Postler A, Lützner C, Beyer F, Tille E, Lützner J. Analysis of Total Knee Arthroplasty revision causes. BMC Musculoskelet Disord. 2018 Feb 14;19(1):55. doi: 10.1186/s12891-018-1977-y. PMID: 29444666; PMCID: PMC5813428.
13. Kamath AF, Ong KL, Lau E, Chan V, Vail TP, Rubash HE, et al. Quantifying the burden of revision total joint arthroplasty for periprosthetic infection. J Arthroplasty 2015;30:1492-7.

14. Bjerke-Kroll BT, Christ AB, McLawhorn AS, Sculco PK, JulesElysée KM, Sculco TP. Periprosthetic joint infections treated with two-stage revision over 14 years: an evolving microbiology profile. J Arthroplasty 2014;29:877-82.

15. The New Zealand Joint Registry Eighteen Year Report January 1999 to December 2016, 2018

16. Canadian Institute for Health Information. Hip and Knee Replacements in Canada, 2017-2018: Canadian Joint Replacement Registry Annual Report. Ottawa, ON: CIHI; 2019.

17. Springer BD, Cahue S, Etkin CD, Lewallen DG, McGrory BJ. Infection burden in total hip and knee arthroplasties: an international registry-based perspective. Arthroplast Today. 2017;3(2):137-140. Published 2017 Jun 20. doi:10.1016/j.artd.2017.05.003

18. Pirruccio K, Premkumar A, Sheth NP. The burden of prosthetic hip dislocations in the United States is projected to significantly increase by 2035. HIP International. May 2020.
Have you read the current trends in Medical and Health Research in the Philippines?

Acta Medica Philippina Access Online: www.actamedicaphilippina.upm.edu.ph 